\title{
Different source of sample strains corresponds to different characteristics of patients with non- tuberculous mycobacterium.
}

\section{Xinling Pan ( $\sim$ panfengyuwuzu@163.com )}

Affiliated Dongyang Hospital of Wenzhou Medical University

Jin Zhao

Affiliated Dongyang Hospital of Wenzhou Medical University

Xiaoling Jin

Affiliated Dongyang Hospital of Wenzhou Medical University

Songjun Ji

Affiliated Dongyang Hospital of Wenzhou Medical University

\section{Research article}

Keywords: non-tuberculous mycobacteria, chronic obstructive pulmonary disease, bronchiectasis, sputum, bronchoalveolar lavage fluid, sample source

Posted Date: July 14th, 2020

DOI: https://doi.org/10.21203/rs.3.rs-41183/v1

License: (c) (i) This work is licensed under a Creative Commons Attribution 4.0 International License. Read Full License 


\section{Abstract}

Background: The non-tuberculous mycobacteria (NTM) strains are important in the diagnosis of pulmonary diseases. However, the different source of the sample may correspond to different characteristics of both NTM disease and infected patients and these aspects have not been investigated.

Methods: Samples isolated from different sources in inpatients with NTM strains were comprehensively analyzed in this study, including basic information, laboratory examination and coexisting diseases.

Results: A total of 242 inpatients with NTM strains were considered in this study. Sputum $(n=129)$ and bronchoalveolar lavage fluid (BALF, $n=50$ ) were the most common samples among patients with NTM strains from whom a sample was collected from a single source $(n=179)$. The patients with NTM-positive BALF differed in age, gender, acid fast assay, the percentage of presence of chronic obstructive pulmonary disease (COPD) and bronchiectasis, and symptom duration compared to the patients with NTM-positive sputum. The patients with COPD were older and mainly male, with longer symptom duration, with higher rates of smoking history and prostatic hyperplasia than those without COPD. Patients with bronchiectasis were mainly females, with a lower percentage of them with a tuberculosis history and smoking history compared to patients without bronchiectasis. Excluding the patients with COPD and bronchiectasis, patients with NTM-positive sputum were older, with positive results in acid-fast assay and T. spot assay compared to patients with NTM-positive BALF.

Conclusions: The different specimen source in NTM patients was associated with different patients' characteristics, and its choice could be influenced by presence of COPD and bronchiectasis. Patients with COPD and bronchiectasis differed in gender and smoking history, thus, these aspects should be comprehensively analyzed during the investigation of patients with pulmonary NTM infection.

\section{Background}

Non-tuberculous mycobacterium (NTM) causes infection in immunocompetent individuals and even in children, contradicting the traditional knowledge that NTM diseases occur only in an immunosuppressed population [1-3]. The significance of NTM isolation in developing countries has not been adequately considered due to the poor evidence of human-to-human transmission (except for Mycobacterium abscesses among patients with cystic fibrosis) [2, 4]. Although a clear association between NTM strains and diseases has not been clarified, increasing evidence showed that patients with NTM show a higher mortality than that without NTM $[5,6]$.

Based on the principles proposed by the ATS/IDSA statement, the diagnosis of pulmonary NTM (especially Mycobacterium avium complex) needs at least two sputum culture or one bronchoalveolar lavage fluid (BALF) culture, thus, repeated isolation of NTM in clinical specimen may related to clinical significance[7]. Our previous study showed that the Mycobacterium avium complex is the most common NTM in Dongyang, located in the middle of Zhejiang Province, similar to other regions [8, 9], indicating the urgent need to establish the relationship between isolated strains and clinical features of patients. 
Previous studies showed that the repeated collection and isolation of NTM strains from patients was closely associated with a higher percentage of COPD when compared to the percentage revealed by a single isolation $[10,11]$. However, both studies above did not evaluate the correspondence of different source of the sample with different characteristics of both NTM disease and infected patients.

BALF is superior to the expectorated sputum in the diagnosis of lower respiratory tract tuberculosis [12, 13]. Indeed in patients with suspected sputum-smear-negative pulmonary tuberculosis, Liu et., al, found that the sensitivity of $63.4 \%$ by culture or nucleic acid amplification test from BALF was higher than the sensitivity of $43.5 \%$ from sputum[13]. In addition, the presence of the Mycobacterium tuberculosis complex (MTBC) was detected in BALF specimen from patients who could no produce sputum. In addition, adenosine deaminase activity in BALF is useful in the diagnosis of tuberculosis with negative sputum smears [14]. The authors found that, among all the collected samples, BALF samples positive for MTBC is associated with patients' characteristics including younger age, existence of pulmonary cavity and positive interferon-gamma release assay [13]. As suggested by studies on tuberculosis, the pathogens from various specimen sources may not only differ in susceptibility and specificity in smear or culture assay for diagnosis, but also reflect different risk factors for the host. However, few data are available regarding the association between specimen source and characteristics of patients with NTM strains.

Therefore, in this study, the clinical records of patients with NTM who were admitted to our hospital were retrospectively analyzed. The specimen source where the NTM strain was isolated was the key point we focused on and the potential association with the characteristics of patients was comprehensively analyzed.

\section{Materials And Methods}

\section{Clinical data reviewing and collection}

The clinical records of inpatients and the corresponding NTM isolates were collected as previously described and anonymously reviewed [10]. The term "NTM isolates" is referred only to positive NTM isolates due to the aim of this work. Beside 204 cases collected in a previous work, 69 newly added cases were included. The inpatients were admitted to the hospital from Jan 2016 to Dec 2019. After excluding the repeated cases and those who may be infected by MTBC as assessed by Gene Xpert assay results, a final number of 242 patients was involved in this study (Fig. 1A). The sample sources have never been analyzed in patient with NTM strains, then, they were reviewed for the first time in this study after receiving detailed information from physicians. Coexisting diseases apart from the diagnosed diseases were considered when the inpatient was discharged. According to the sample source where NTM were isolated, all patients were divided into patients with sputum positive culture (despite the number of times the sample was collected) and patients with BALF positive culture, excluding those with both sputum and BALF positive. 


\section{Statistical analysis}

Statistical analysis was performed using SPSS software 23.0 version (IBM, USA). All data from the inpatients were categorized into different groups and the differences were analyzed by $\chi^{2}$ test or fisher exact test. A $p$ value less than 0.05 was considered statistically significant.

\section{Results}

\section{Distribution of different specimen sources in this study}

A total of 274 NTM strains were identified by MPB64 assay performed in the laboratory of the hospital where the patients were admitted. After excluding the repeated samples from the same patients and patients without available clinical records, 242 inpatients were finally analyzed for specimen sources in this study (Fig. 1B). Among them, 179 were subjected to only one type of sample collection (sputum or BALF): 116 (47.9\%) cases were positive after testing a single sputum; 13 cases (5.4\%) were positive after testing multiple sputum; 50 (20.7\%) cases were BALF positive. Forty-three cases were subjected to both sputum and BALF culture: 23 (9.5\%) cases were sputum negative and BLAF positive; 7 (2.9\%) cases were sputum positive and BLAF negative; $13(5.4 \%)$ cases were sputum positive and BLAF positive. The remaining 20 (8.3\%) cases were subjected to other types of sample collection, such as urine, cerebrospinal fluid, hydrothorax and pus.

\section{Difference in characteristics between patients with sputum and BALF positive results}

Considering that the sample isolation source was significantly important in the diagnosis of pulmonary NTM, the differences between patients with sputum isolation (including single sputum positive, multiple sputum positive, sputum positive with BALF negative) and BALF isolation (including BALF positive, sputum negative with BALF positive) were analyzed (Table 1). Patients with BALF NTM positive samples were significantly younger that those with sputum NTM positive samples $(63.8 \pm 10.3, p<0.001)$, but a significantly higher percentage of female patients were present among them $(57.5 \%, p=0.008)$. Among the laboratory assay results used for tuberculosis, the patients with BALF NTM positive samples showed a significantly lower frequency of positive acid-fast staining results $(p=0.02)$ compared to patients with sputum NTM positive samples. In addition, patients with sputum NTM positive samples were mainly accompanied with COPD $(p<0.001)$ and hypertension $(p=0.003)$, but the percentage of bronchiectasis was lower in these patients $(p=0.002)$ compared to patients with BALF NTM positive samples. Radical imaging results (nodular or cavity manifestation) showed no significant difference between the patients belonging to the sputum and BALF isolation groups. The duration of the symptoms was significantly shorter in patients with BALF NTM positive samples compared to patients with sputum NTM positive samples, with $39.7 \%$ of cases with symptoms duration longer than one year $(p=0.005)$. 
Table 1

Characteristics of patients with NTM from different specimen sources.

\begin{tabular}{|c|c|c|c|c|}
\hline Features & values & Sputum $(n=136)$ & BALF $(n=73)$ & $p$ value \\
\hline Age $($ mean $\pm S D)$ & & $73.0 \pm 13.2$ & $63.8 \pm 10.3$ & $<0.0001$ \\
\hline \multirow[t]{2}{*}{ Gender } & Female & $52(38.2)$ & $42(57.5)$ & $0.008 a$ \\
\hline & Male & $84(61.8)$ & $31(42.5)$ & \\
\hline \multirow[t]{2}{*}{ Occupation } & Farmer & $109(80.1)$ & $63(86.3)$ & $0.266 a$ \\
\hline & Non-farmer & $27(19.9)$ & $10(13.7)$ & \\
\hline \multirow[t]{2}{*}{ Smoking history (male) } & Yes & $55(65.5)$ & $19(61.3)$ & $0.678 b$ \\
\hline & No & $29(34.5)$ & $12(38.7)$ & \\
\hline \multirow[t]{2}{*}{ TB history } & Yes & $33(24.3)$ & $16(21.9)$ & $0.703 a$ \\
\hline & No & $103(75.7)$ & $57(78.1)$ & \\
\hline \multirow[t]{2}{*}{ Surgeon history } & Yes & $61(44.9)$ & $24(32.9)$ & $0.093 a$ \\
\hline & No & $75(55.1)$ & $49(67.1)$ & \\
\hline \multirow[t]{2}{*}{ CT nodular } & Nodular & $62(47.3)$ & $37(56.9)$ & $0.206 a$ \\
\hline & Non-nodular & $69(52.7)$ & $28(43.1)$ & \\
\hline \multirow[t]{2}{*}{ CT cavity } & Yes & $14(10.7)$ & $6(9.2)$ & $0.751 a$ \\
\hline & No & $117(89.3)$ & $59(90.8)$ & \\
\hline \multirow[t]{2}{*}{ TB-Antibody } & Positive & $22(19.6)$ & $7(11.7)$ & $0.183 a$ \\
\hline & Negative & $90(80.4)$ & $53(88.3)$ & \\
\hline \multirow[t]{2}{*}{ Acid fast assay } & Positive & $38(29.0)$ & $7(9.9)$ & $0.002 a$ \\
\hline & Negative & $93(71.0)$ & $64(90.1)$ & \\
\hline \multirow[t]{2}{*}{ T. spot assay } & Positive & $17(38.6)$ & $6(18.8)$ & $0.062 a$ \\
\hline & Negative & $27(61.4)$ & $26(81.3)$ & \\
\hline \multirow[t]{2}{*}{ COPD } & Yes & $66(48.5)$ & $13(17.8)$ & $<0.001 \mathrm{a}$ \\
\hline & No & $70(51.5)$ & $60(82.2)$ & \\
\hline \multirow[t]{2}{*}{ Bronchiectasis } & Yes & $25(18.4)$ & $28(38.4)$ & $0.002 a$ \\
\hline & No & $111(81.6)$ & $45(61.6)$ & \\
\hline Diabetes & Yes & $9(6.6)$ & $4(5.5)$ & $0.745 a$ \\
\hline
\end{tabular}




\begin{tabular}{|lllll|}
\hline Features & values & Sputum $(\mathbf{n = 1 3 6 )}$ & BALF $(\mathbf{n}=73)$ & $\boldsymbol{p}$ value \\
\hline \multirow{2}{*}{ Tumor } & No & $127(93.4)$ & $68(94.5)$ & \\
& Yes & $12(8.8)$ & $5(6.8)$ & $0.619 \mathrm{a}$ \\
\cline { 2 - 4 } & No & $124(91.2)$ & $68(93.2)$ & \\
Prostatic hyperplasia(male) & Yes & $51(60.7)$ & $14(45.2)$ & $0.135 \mathrm{a}$ \\
\hline Duration of symptoms & No & $33(39.3)$ & $17(54.8)$ & \\
\hline & $\leq 1$ year & $54(39.7)$ & $44(60.3)$ & $0.005 a$ \\
\hline a Pearson Chi-Square. b Fisher's Exact Test. c Continuity Correction. The bold $p$ value was significant. \\
\hline
\end{tabular}

\section{Coexistence of COPD or bronchiectasis in patients with NTM isolation}

As suggested by the results above, the specimen sources were associated with coexisting diseases including COPD and bronchiectasis. The influence of COPD on the characteristics of patients with NTM was analyzed (Table 2). Patients with COPD were older and more frequently represented by males compared to patients without COPD. Excluding the female patients, the percentage of patients with a smoking history was higher in COPD patients than that in patients without COPD $(53.7 \%$ vs $79.2 \%, p=$ 0.004). In male individuals, prostatic hyperplasia was present in $79.2 \%$ of patients with COPD compared to the $37.3 \%$ in patients without COPD $(p<0.001)$. The $85 \%$ of patients with COPD had a symptom duration longer than one year compared with the patients without COPD $(34.2 \%)(p<0.001)$. 
Table 2

Influence of COPD on characteristics of patients with NTM.

\begin{tabular}{|c|c|c|c|c|}
\hline Features & values & without COPD $(n=142)$ & $\begin{array}{l}\text { with } \\
\text { COPD }(n=80)\end{array}$ & $p$ value \\
\hline Age $($ mean \pm SD) & & $66.1 \pm 14.0$ & $75.2 \pm 8.1$ & $<0.0001$ \\
\hline \multirow[t]{2}{*}{ Gender } & Female & $75(52.8)$ & $27(33.8)$ & $0.006 a$ \\
\hline & Male & $67(47.2)$ & $53(66.3)$ & \\
\hline \multirow[t]{2}{*}{ Occupation } & Farmer & $121(85.2)$ & $63(78.8)$ & $0.22 a$ \\
\hline & Non-farmer & $21(14.8)$ & $17(21.3)$ & \\
\hline \multirow[t]{2}{*}{ Smoking history (male) } & Yes & $36(53.7)$ & $42(79.2)$ & $0.004 a$ \\
\hline & No & $31(46.3)$ & $11(20.8)$ & \\
\hline \multirow[t]{2}{*}{ TB history } & Yes & $29(20.4)$ & $21(26.3)$ & $0.318 a$ \\
\hline & No & $113(79.6)$ & $59(73.8)$ & \\
\hline \multirow[t]{2}{*}{ Surgeon history } & Yes & $53(37.3)$ & $38(47.5)$ & $0.139 a$ \\
\hline & No & $89(62.7)$ & $42(52.5)$ & \\
\hline \multirow[t]{2}{*}{ CT nodular } & Nodular & $71(53.0)$ & $37(49.3)$ & $0.612 a$ \\
\hline & Non-nodular & $63(47.0)$ & $38(50.7)$ & \\
\hline \multirow[t]{2}{*}{ CT cavity } & Yes & $18(13.4)$ & $5(6.7)$ & $0.134 a$ \\
\hline & No & $116(86.6)$ & $70(93.3)$ & \\
\hline \multirow[t]{2}{*}{ TB-Antibody } & Positive & $21(17.6)$ & $10(15.2)$ & $0.663 a$ \\
\hline & Negative & $98(82.4)$ & $56(84.8)$ & \\
\hline \multirow[t]{2}{*}{ Acid fast assay } & Positive & $29(21.3)$ & $20(25.3)$ & $0.501 a$ \\
\hline & Negative & 107 (78.7) & $59(74.7)$ & \\
\hline \multirow[t]{2}{*}{ T. spot assay } & Positive & $20(31.7)$ & 5 (21.7) & $0.366 a$ \\
\hline & Negative & $43(68.3)$ & 18 (78.3) & \\
\hline \multirow[t]{2}{*}{ Bronchiectasis } & Yes & $41(28.9)$ & $16(20.0)$ & $0.146 a$ \\
\hline & No & 101 (71.1) & $64(80.0)$ & \\
\hline \multirow[t]{2}{*}{ Diabetes } & Yes & $10(7.0)$ & $5(6.3)$ & $0.821 a$ \\
\hline & No & $132(93.0)$ & 75 (93.8) & \\
\hline
\end{tabular}

a Pearson Chi-Square. b Fisher's Exact Test. c Continuity Correction. The bold $p$ value was significant. 


\begin{tabular}{|c|c|c|c|c|}
\hline Features & values & without COPD $(n=142)$ & $\begin{array}{l}\text { with } \\
\text { COPD }(n=80)\end{array}$ & $p$ value \\
\hline \multirow[t]{2}{*}{ Tumor } & Yes & $12(8.5)$ & $6(7.5)$ & $0.803 a$ \\
\hline & No & 130 (91.5) & $74(92.5)$ & \\
\hline \multirow[t]{2}{*}{ Prostatic hyperplasia (male) } & Yes & $25(37.3)$ & $42(79.2)$ & $<0.0001 a$ \\
\hline & No & $42(62.7)$ & $11(20.8)$ & \\
\hline \multirow[t]{2}{*}{ Duration of symptoms } & $\leq 1$ year & $96(67.6)$ & $12(15.0)$ & $<0.0001 a$ \\
\hline & $>1$ year & $46(32.4)$ & $68(85.0)$ & \\
\hline
\end{tabular}

Furthermore, the presence of bronchiectasis influenced the presence of NTM depending on the characteristics of the patients (Table 3 ). The $77.2 \%$ of patients with bronchiectasis were female, significantly higher than the $35.2 \%$ of patients without bronchiectasis $(p<0.001)$. Among male NTM patients, the percentage of them with smoking history was lower in the ones with bronchiectasis than that in the ones without bronchiectasis $(23.1 \%$ vs $70.1 \%, p=0.002)$. The percentage of patients with tuberculosis history was $35.1 \%$ in the ones with bronchiectasis, while it was $18.2 \%$ in the ones without bronchiectasis $(p=0.008)$. 
Table 3

Influence of bronchiectasis on characteristics of patients with NTM.

\begin{tabular}{|c|c|c|c|c|}
\hline Features & values & $\begin{array}{l}\text { Without Bronchiectasis } \\
(n=165)\end{array}$ & $\begin{array}{l}\text { With Bronchiectasis } \\
(n=57)\end{array}$ & $p$ value \\
\hline Age $($ mean $\pm S D)$ & & $69.7 \pm 13.4$ & $68.7 \pm 11.5$ & 0.693 \\
\hline \multirow[t]{2}{*}{ Gender } & Female & 58 (35.2) & $44(77.2)$ & ¿.0001a \\
\hline & Male & 107 (64.8) & $13(22.8)$ & \\
\hline \multirow[t]{2}{*}{ Occupation } & Farmer & 135 (81.8) & $49(86.0)$ & $0.474 a$ \\
\hline & $\begin{array}{l}\text { Non- } \\
\text { farmer }\end{array}$ & $30(18.2)$ & $8(14.0)$ & \\
\hline \multirow{2}{*}{$\begin{array}{l}\text { Smoking history } \\
\text { (male) }\end{array}$} & Yes & 75 (70.1) & $3(23.1)$ & $0.002 c$ \\
\hline & No & 32 (29.9) & $10(76.9)$ & \\
\hline \multirow[t]{2}{*}{ TB history } & Yes & 30 (18.2) & $20(35.1)$ & $0.008 a$ \\
\hline & No & 135 (81.8) & $37(64.9)$ & \\
\hline \multirow[t]{2}{*}{ Surgeon history } & Yes & 69 (41.8) & $22(38.6)$ & $0.67 a$ \\
\hline & No & $96(58.2)$ & 35 (61.4) & \\
\hline \multirow[t]{2}{*}{ Acid fast assay } & Positive & 34 (21.3) & 15 (27.3) & $0.358 a$ \\
\hline & Negative & $126(78.8)$ & $40(72.7)$ & \\
\hline \multirow[t]{2}{*}{ CT nodular } & Nodular & $83(52.5)$ & $25(49.0)$ & $0.663 a$ \\
\hline & $\begin{array}{l}\text { Non- } \\
\text { nodular }\end{array}$ & 75 (47.5) & $26(51.0)$ & \\
\hline \multirow[t]{2}{*}{ CT cavity } & Yes & 18 (11.4) & $5(9.8)$ & $0.753 a$ \\
\hline & No & 140 (88.6) & $46(90.2)$ & \\
\hline \multirow[t]{2}{*}{ TB-Antibody } & Positive & 25 (17.9) & $6(13.3)$ & $0.48 a$ \\
\hline & Negative & 115 (82.1) & 39 (86.7) & \\
\hline \multirow[t]{2}{*}{ T. spot assay } & Positive & $23(32.9)$ & $2(12.5)$ & $0.189 a$ \\
\hline & Negative & $47(67.1)$ & $14(87.5)$ & \\
\hline \multirow[t]{2}{*}{ COPD } & Yes & $64(38.8)$ & $16(28.1)$ & $0.146 a$ \\
\hline & No & $101(61.2)$ & 41 (71.9) & \\
\hline
\end{tabular}

a Pearson Chi-Square. b Fisher's Exact Test. c Continuity Correction. The bold $p$ value was significant. 


\begin{tabular}{|lllll|}
\hline Features & values & $\begin{array}{l}\text { Without Bronchiectasis } \\
(\mathbf{n = 1 6 5 )}\end{array}$ & $\begin{array}{l}\text { With Bronchiectasis } \\
(\mathbf{n = 5 7})\end{array}$ & $p$ value \\
\hline Diabetes & Yes & $13(7.9)$ & $2(3.5)$ & $0.408 \mathrm{c}$ \\
\hline No & $152(92.1)$ & $55(96.5)$ & $0.528 \mathrm{c}$ \\
\hline $\begin{array}{l}\text { Prostatic hyperplasia } \\
\text { (male) }\end{array}$ & Yes & $15(9.1)$ & $3(5.3)$ & $0.661 \mathrm{a}$ \\
\hline & No & $150(90.9)$ & $54(94.7)$ & $0.146 \mathrm{a}$ \\
\hline $\begin{array}{l}\text { Duration of } \\
\text { symptoms }\end{array}$ & $59(55.1)$ & $8(61.5)$ & \\
\hline \multicolumn{4}{|l}{} \\
\hline
\end{tabular}

\section{Impact of sample source on the characteristics of patients with positive NTM isolation excluding the presence of coexisting diseases}

To investigate the actual influence of specimen resources on patients with NTM, the patients with COPD and bronchiectasis were excluded in our further analysis (Table 4). The patients with NTM strains from sputum samples were older than those with NTM strains from BALF $(62.4 \%$ vs $68.8 \%, p=0.034)$. Acid fast assay (performed in the laboratory of the hospital where the patients were admitted) in sputum specimen showed a higher percentage of positive results than that in BALF specimen $(25.9 \%$ vs $5.7 \%, p=$ 0.015). Besides, $14.3 \%$ of patients with NTM from BALF displayed positive result in T. spot assay, lower than $51.7 \%$ in those with NTM from sputum $(p=0.019)$. 
Table 4

The influence of specimen sources on patients with NTM isolates but without COPD or Bronchiectasis.

\begin{tabular}{|c|c|c|c|c|}
\hline Features & values & Sputum $(n=57)$ & BALF $(n=36)$ & $p$ value \\
\hline Age $($ mean $\pm S D)$ & & $68.8 \pm 17.4$ & $62.4 \pm 11.2$ & 0.0340 \\
\hline \multirow[t]{2}{*}{ Gender } & Female & $21(36.8)$ & $19(52.8)$ & $0.131 a$ \\
\hline & male & $36(63.2)$ & $17(47.2)$ & \\
\hline \multirow[t]{2}{*}{ Occupation } & Farmer & $49(86.0)$ & $29(80.6)$ & $0.49 a$ \\
\hline & Non-farmer & $8(14.0)$ & $7(19.4)$ & \\
\hline \multirow[t]{2}{*}{ Smoking history (male) } & Yes & $20(55.6)$ & $11(64.7)$ & $0.528 a$ \\
\hline & No & $16(44.4)$ & $6(35.3)$ & \\
\hline \multirow[t]{2}{*}{ TB history } & Yes & $11(19.3)$ & $4(11.1)$ & $0.296 a$ \\
\hline & No & $46(80.7)$ & $32(88.9)$ & \\
\hline \multirow[t]{2}{*}{ Surgeon history } & Yes & $23(40.4)$ & $12(33.3)$ & $0.496 a$ \\
\hline & No & $34(59.6)$ & $24(66.7)$ & \\
\hline \multirow[t]{2}{*}{ CT nodular } & Nodular & $30(53.6)$ & $17(51.5)$ & 0.851 \\
\hline & Non-nodular & $26(46.4)$ & $16(48.5)$ & \\
\hline \multirow[t]{2}{*}{ CT cavity } & Yes & $7(12.5)$ & $3(9.1)$ & $0.885 c$ \\
\hline & No & $49(87.5)$ & $30(90.9)$ & \\
\hline \multirow[t]{2}{*}{ TB-Antibody } & Positive & $13(26.5)$ & $3(11.1)$ & $0.115 a$ \\
\hline & Negative & $36(73.5)$ & $24(88.9)$ & \\
\hline \multirow[t]{2}{*}{ Acid fast assay } & Positive & $14(25.9)$ & $2(5.7)$ & $0.015 a$ \\
\hline & Negative & $40(74.1)$ & $33(94.3)$ & \\
\hline \multirow[t]{2}{*}{ T. spot assay } & Positive & $15(51.7)$ & $2(14.3)$ & $0.019 a$ \\
\hline & Negative & $14(48.3)$ & $12(85.7)$ & \\
\hline \multirow[t]{2}{*}{ Diabetes } & Yes & $6(10.5)$ & $1(2.8)$ & $0.329 c$ \\
\hline & No & $51(89.5)$ & 35 (97.2) & \\
\hline \multirow[t]{2}{*}{ Tumor } & Yes & $7(12.3)$ & $3(8.3)$ & $0.799 c$ \\
\hline & No & $50(87.7)$ & $33(91.7)$ & \\
\hline
\end{tabular}

a, Pearson Chi-Square. b, Fisher's Exact Test. c, Continuity Correction. The bold $p$ value was significant. 


\begin{tabular}{|lllll|}
\hline Features & values & Sputum(n= 57) & BALF $(\mathbf{n = 3 6 )}$ & p value \\
\hline Prostatic hyperplasia (male) & Yes & $12(33.3)$ & $7(41.2)$ & $0.578 \mathrm{a}$ \\
\hline Duration of symptoms & No & $24(66.7)$ & $24(66.7)$ & \\
\hline & $>1$ year & $40(70.2)$ & $28(77.8)$ & $0.421 \mathrm{a}$ \\
\hline $\begin{array}{l}\text { a, Pearson Chi-Square. b, Fisher's Exact Test. c, Continuity Correction. The bold } p \text { value was } \\
\text { significant. }\end{array}$ & & & \\
\hline
\end{tabular}

\section{Discussion}

Different sample source indicated different colonization or infection sites, which might be significant in the diagnosis of infectious disease such as NTM infection, since it exists in living environment. Therefore, the patients with NTM isolates from different sample sources mainly including sputum and BALF were retrospectively analyzed in this study. Our results showed that the sample source was associated with different gender, age, acid fast assay, T. spot assay, COPD and bronchiectasis among NTM patients

Sputum and BALF are common samples collected for the diagnosis of respiratory infectious disease. Expectorated sputum is easier to collect, but it is more likely contaminated by environmental factor. The BALF is collected from the lower respiratory tract and free of contamination from mouth, but it is only available in patients who are subjected to bronchoscopy and it is forbidden in some patients with severe respiratory diseases. As suggested in the principles of diagnosis of pulmonary NTM tuberculous by ATS/IDSA, repeated positive culture from sputum sample or single positive culture from BALF is an indication of the presence of NTM [7]. Our results showed that the patients with sputum positive for NTM strain were older than those with BALF positive, suggesting that bronchoscopy might be more tolerated by younger inpatient as they were usually hospitalized because of suspected infection accompanied with coexisting mild diseases. Indeed, coexisting diseases influence the choice in the selection of the sample source. As suggested by our study, BALF positive samples were closely associated with patients with bronchiectasis and sputum positive samples were associated with patients with COPD. In clinical practice, when bronchiectasis is suspected, a bronchoscopy test is suggested, while patients with COPD are not subjected to bronchoscopy especially when they suffer from exacerbation [15].

The association between COPD and pulmonary NTM disease has been increasingly reported, but whether NTM infection increases the risk of COPD or COPD results in high risk of NTM infection has not been clearly clarified $[2,16-19]$. Patients with COPD are more susceptible to NTM infection than those with other chronic pulmonary diseases [20,21] (except cystic fibrosis), and inhaled corticosteroids increase the risk of NTM infection [19]. Chronic inflammation and airway remodeling may contribute to higher susceptibility to NTM infection. Among patients with NTM in this study, the patients with COPD were mainly older and males, as it was also previously reported [11]. Limited data are available regarding the influence of NTM infection on COPD, but our previous work and another study suggested that repeated 
isolation of samples in patients was associated with a higher percentage of COPD $[10,11]$. In a follow up study, COPD patients with NTM-positive sample more likely experience exacerbation than those with NTM-negative samples, and patients with multiple positive samples had a more pronounced pulmonary function decline than those with single or no positive samples [22]. In addition, our results showed that male patients with COPD had an increased risk of prostatic hyperplasia than those without COPD, which could be explained by the fact that male patients with COPD are at higher risk of benign prostatic hyperplasia despite of the presence of NTM $[23,24]$. The relationship between COPD and prostatic hyperplasia might be explained by the physical inactivity and chronic inflammation $[25,26]$.

Nodular-related bronchiectasis is of significance in the diagnosis and treatment of pulmonary NTM infection. Although bronchiectasis has been observed in $20 \%-30 \%$ of patients with NTM, NTM infection seems not common in patients with bronchiectasis $[10,27]$. Indeed, $34-50 \%$ of patients with multiple small nodules (and sometimes cavity or cavities) combined with their diffusion (or widespread) indicate the presence of active pulmonary NTM infection [28]. In our previous study, bronchiectasis was observed mainly in female patients with NTM, result that is similar to another study from Singapore [11]. Among patients with NTM, the percentage of patients with tuberculosis history was higher in the ones with bronchiectasis than that in patients without bronchiectasis, meaning that pulmonary tuberculosis might result in anatomic changes of the respiratory tract and reinfection by other pathogens such NTM [29]. The percentage of tuberculosis history between patients with COPD or without COPD was not significantly different, suggesting that previous tuberculosis infection might not be associated with the pathogenesis of COPD. Previous works showed that the percentage of patients with bronchiectasis presenting multiple NTM positive isolations is comparable to the percentage of patients with bronchiectasis presenting a single NTM positive isolation $[10,11]$, indicating the limited impact of NTM infection on bronchiectasis. Instead, repeated isolations of NTM positive strains partly contributes to the progression of COPD $[10,11]$.

After excluding patients with COPD and bronchiectasis, sputum resource from patients with positive NTM was associated with age, acid fast assay and T. spot assay. Higher percentage of positive result in acidfast assay could be explained by the fact that the repeated sample collection was more common when sputum was collected than when BALF was collected. The higher percentage of positive results of T. spot assay in patients with positive sputum than that in patients with positive BALF might represent a warning that this result could be more likely misdiagnosed as MTBC infection before species identification.

\section{Conclusions}

Samples positive for NTM strains were associated with age, gender, acid fast assay, duration of symptom and pre-existing diseases. Co-existence of COPD in patients with NTM isolation was related to age, gender, smoking history, duration of symptom and prostatic hyperplasia. Presence of bronchiectasis was influenced by age, gender, tuberculosis history and smoking history. The characteristics of patients with NTM were complicated by coexisting diseases especially COPD and bronchiectasis and should be 
comprehensively analyzed, which could be of significance in the prognosis and treatment of pulmonary NTM diseases.

\section{Abbreviations}

NTM, non-tuberculous mycobacteria; COPD, chronic obstructive pulmonary disease, BALF, bronchoalveolar lavage fluid; TB, tuberculosis; MTBC, mycobacterium tuberculosis complex.

\section{Declarations}

\section{Ethics approval and consent to participate:}

Verbal informed consent from participants was obtained considering that no identifiable individual information was displayed in research. This work was approved by the Ethics Committee of Dongyang People's Hospital Ethics Committee and Institutional Review Board.

\section{Consent for publication:}

Not applicable.

\section{Availability of data and materials:}

All data generated or analyzed during this study are included in this published article.

\section{Competing interests:}

The authors declare that they have no competing interests.

\section{Funding:}

The design of the study and data collection were supported by Natural Science Foundation of Zhejiang Province (LQ19C010001). The analysis, and interpretation of data and manuscript writing was funded by the National Natural Science Foundation of China (Grant No. 81902109).

\section{Authors' contributions:}

PX designed this study, analyzed the data and wrote this manuscript. JS collected the clinical data including the laboratory examination results. JX participated in data confirmation and collection of data from clinical records. ZJ was involved in data collection and analysis. All authors read and approved the final manuscript. 


\section{Acknowledgements:}

Not applicable

\section{References}

1. Vandenbos F, Marcq L, Novellas S, Chyderiotis G, Haudebourg J, Benchetrit M, et al. The presence of mycobacteria in bronchoalveolar lavage fluid from an immunocompetent patient does not necessarily imply tuberculosis. Ann Pathol. 2009;29:504-6.

2. Koh WJ. Nontuberculous Mycobacteria-Overview. Microbiol Spectr. 2017; 5.

3. Varghese B, Enani M, Shoukri M, AlJohani S, AlThawadi S, Al-Hajoj S. Burden of non-tuberculous mycobacterial diseases in Saudi Arabian children: The first nationwide experience. J Infect Public Health. 2019;12:803-8.

4. Horne D, Skerrett S. Recent advances in nontuberculous mycobacterial lung infections. F1000Res. 2019; 8.

5. Marras TK, Campitelli MA, Lu H, Chung H, Brode SK, Marchand-Austin A, et al. Pulmonary Nontuberculous Mycobacteria-Associated Deaths, Ontario, Canada, 2001-2013. Emerg Infect Dis. 2017;23:468-76.

6. Marras TK, Vinnard C, Zhang Q, Hamilton K, Adjemian J, Eagle G, et al. Relative risk of all-cause mortality in patients with nontuberculous mycobacterial lung disease in a US managed care population. Respir Med. 2018;145:80-8.

7. Griffith DE, Aksamit T, Brown-Elliott BA, Catanzaro A, Daley C, Gordin F, et al. An official ATS/IDSA statement: diagnosis, treatment, and prevention of nontuberculous mycobacterial diseases. Am J Respir Crit Care Med. 2007;175:367-416.

8. Hoefsloot W, van Ingen J, Andrejak C, Angeby K, Bauriaud R, Bemer P, et al. The geographic diversity of nontuberculous mycobacteria isolated from pulmonary samples: an NTM-NET collaborative study. Eur Respir J. 2013;42:1604-13.

9. Pan X, Zhou Y, Li Z, Zhang J, Hong L, Shi Y, et al. Investigation of non-tuberculous mycobacteria in a primary hospital from southeastern China. J Infect Dev Ctries. 2019;13:1095-100.

10. Ji S, Xu W, Sun J, Shi Y, Pan X. Retrospective analysis of patients with non-tuberculous mycobacteria from a primary hospital in Southeast China. Sci Rep. 2020;10:1060.

11. Zhang ZX, Cherng BPZ, Sng LH, Tan YE. Clinical and microbiological characteristics of nontuberculous mycobacteria diseases in Singapore with a focus on pulmonary disease, 2012-2016. BMC Infect Dis. 2019;19:436.

12. Simsek A, Yapici I, Babalik M, Simsek Z, Kolsuz M. Bronchoscopic diagnostic procedures and microbiological examinations in proving endobronchial tuberculosis. J Bras Pneumol. 2016;42:1915. 
13. Liu X, Hou XF, Gao L, Deng GF, Zhang MX, Deng QY, et al. Indicators for prediction of Mycobacterium tuberculosis positivity detected with bronchoalveolar lavage fluid. Infect Dis Poverty. 2018;7:22.

14. Boonsarngsuk V, Suwannaphong S, Laohavich C. Combination of adenosine deaminase activity and polymerase chain reaction in bronchoalveolar lavage fluid in the diagnosis of smear-negative active pulmonary tuberculosis. Int J Infect Dis. 2012;16:e663-8.

15. Kim V, Aaron SD. What is a COPD exacerbation? Current definitions, pitfalls, challenges and opportunities for improvement. Eur Respir J. 2018; 52.

16. Wassilew N, Hoffmann H, Andrejak C. and Lange C. Pulmonary Disease Caused by Non-Tuberculous Mycobacteria. Respiration. 2016;91:386-402.

17. Stout JE, Koh WJ, Yew WW. Update on pulmonary disease due to non-tuberculous mycobacteria. Int $J$ Infect Dis. 2016;45:123-34.

18. Pyarali FF, Schweitzer M, Bagley V, Salamo O, Guerrero A, Sharifi A, et al. Increasing Non-tuberculous Mycobacteria Infections in Veterans With COPD and Association With Increased Risk of Mortality. Front Med (Lausanne). 2018;5:311.

19. Balavoine C, Andrejak C, Marchand-Adam S, Blanc FX. [Relationships between COPD and nontuberculous mycobacteria pulmonary infections]. Rev Mal Respir. 2017;34:1091-7.

20. Winthrop KL, McNelley E, Kendall B, Marshall-Olson A, Morris C, Cassidy M, et al. Pulmonary nontuberculous mycobacterial disease prevalence and clinical features: an emerging public health disease. Am J Respir Crit Care Med. 2010;182:977-82.

21. Char A, Hopkinson NS, Hansell DM, Nicholson AG, Shaw EC, Clark SJ, et al. Evidence of mycobacterial disease in COPD patients with lung volume reduction surgery; the importance of histological assessment of specimens: a cohort study. BMC Pulm Med. 2014;14:124.

22. Huang CT, Tsai YJ, Wu HD, Wang JY, Yu CJ, Lee LN, et al. Impact of non-tuberculous mycobacteria on pulmonary function decline in chronic obstructive pulmonary disease. Int $\mathrm{J}$ Tuberc Lung Dis. 2012;16:539-45.

23. Peng YH, Huang CW, Liao WC, Chen HJ, Yin MC, Huang YM, et al. Association between chronic obstructive pulmonary disease and increased risk of benign prostatic hyperplasia: a retrospective nationwide cohort study. BMJ Open. 2017;7:e015581.

24. Kaplan SA. Re. Association between Chronic Obstructive Pulmonary Disease and Increased Risk of Benign Prostatic Hyperplasia: A Retrospective Nationwide Cohort Study. J Urol. 2018;200:1138-41.

25. Spruit MA, Pitta F, McAuley E, ZuWallack RL. and Nici L. Pulmonary Rehabilitation and Physical Activity in Patients with Chronic Obstructive Pulmonary Disease. Am J Respir Crit Care Med. 2015;192:924-33.

26. Parsons JK, Kashefi C. Physical activity, benign prostatic hyperplasia, and lower urinary tract symptoms. Eur Urol. 2008;53:1228-35.

27. Schweitzer MD, Salamo O, Campos M, Schraufnagel DE, Sadikot R, Mirsaeidi M. Body habitus in patients with and without bronchiectasis and non-tuberculous mycobacteria. PLoS One. 2017;12:e0185095. 
28. Koh WJ, Kwon OJ. Bronchiectasis and non-tuberculous mycobacterial pulmonary infection. Thorax. 2006;61:458. author reply.

29. Hsu D, Irfan M, Jabeen K, Iqbal N, Hasan R, Migliori GB, et al. Post tuberculosis treatment infectious complications. Int J Infect Dis. 2020;92S:41-S5.

\section{Figures}

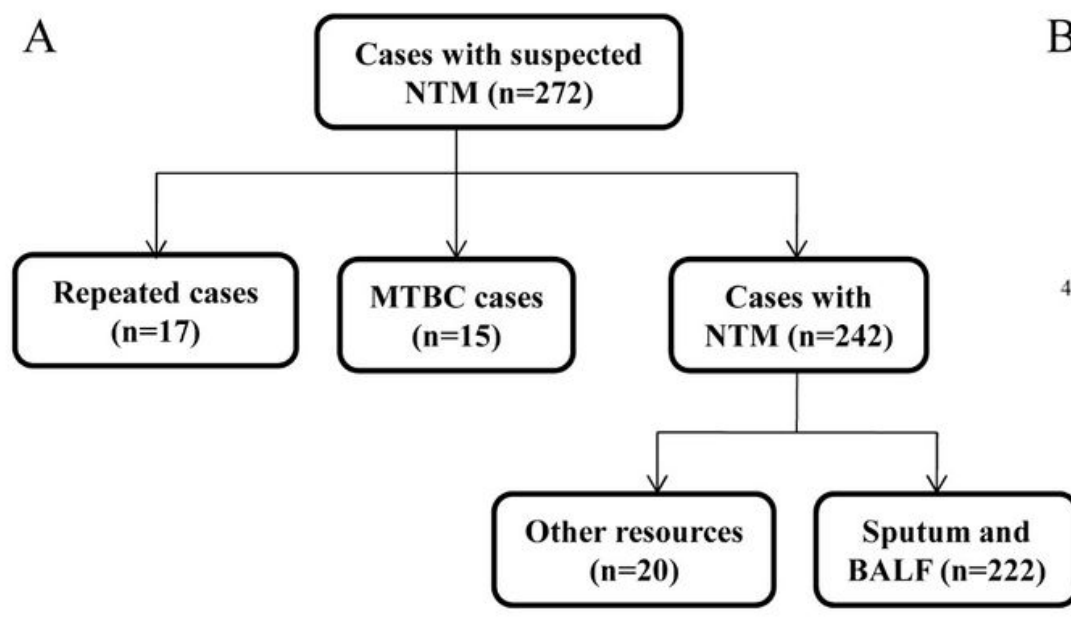

B $5.4 \%$

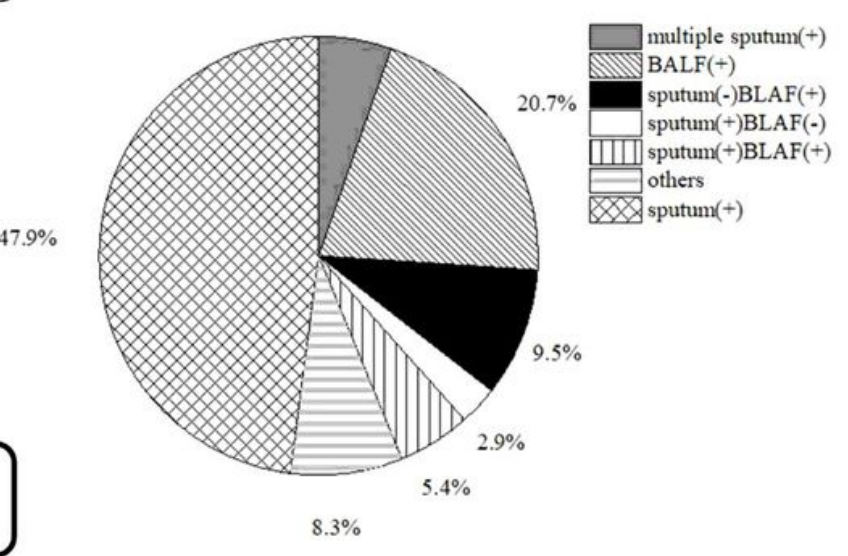

Figure 1

Data collection flow and distribution of sample source of NTM strains. (A) Work flow of data collection; (B) Distribution of sample source of isolated NTM strains. NTM, nontuberculous mycobacteria; MTBC, mycobacterium tuberculosis complex; BALF, bronchoalveolar lavage fluid. 